\title{
Surfaces
}

\section{Suzanne Guellouz. Le dialogue. Coll. Littératures modernes, Presses Universitaires de France, Paris, 1992}

\section{Jean-François Vallée}

Volume 4, 1994

URI : https://id.erudit.org/iderudit/1064976ar

DOI : https://doi.org/10.7202/1064976ar

Aller au sommaire du numéro

Éditeur(s)

Les Presses de l’Université de Montréal

ISSN

1188-2492 (imprimé)

1200-5320 (numérique)

Découvrir la revue

Citer ce compte rendu

Vallée, J.-F. (1994). Compte rendu de [Suzanne Guellouz. Le dialogue. Coll.

Littératures modernes, Presses Universitaires de France, Paris, 1992]. Surfaces,

4. https://doi.org/10.7202/1064976ar d'utilisation que vous pouvez consulter en ligne. 


\section{COMPTE RENDU}

SUZANNE GUELLOUZ: LE DIALOGUE

Jean-François Vallée

Suzanne Guellouz. Le dialogue. Coll. Littératures modernes, Presses Universitaires de France, Paris, 1992.

Il n'existait, jusqu'à tout récemment, aucune monographie française[1] sur la longue tradition littéraire et philosophique du genre dialogué. L'ouvrage de Suzanne Guellouz -- publiée dans la collection $<<$ Littératures modernes $>>$ des Presses universitaires de France[2] -- vise à combler ce vide en nous présentant de manière générale et exhaustive cette forme discursive très particulière, négligée depuis trop longtemps dans les études littéraires.

On comptait déjà, en France, plusieurs études ponctuelles sur les avatars du dialogue à différentes époques : quelques unes sur le dialogue antique[3], moins sur le dialogue médiéval[4], plu sieurs sur le dialogue à la Renaissance[5], ainsi que de nombreuses études des entretiens et dialogues philosophiques des XVIIe et XVIIIe siècles[6]. Il y a bien aussi quelques articles isolés sur le genre dialogué $<<$ en général $>>$ [7], mais aucune étude encore n'avait l'envergure ou l'ambition de l'ouvrage de Guellouz.

Le projet est effectivement ambitieux, car il s'agit d'une tentative d'approche globale -- à la fois théorique et historique -- de ce genre hybride et protéiforme qui a été extrêmement populaire tout au long de l'histoire culturelle de l'Occident (du moins jusqu'au XIXe siècle où il commence à s'étioler). Je ne sais s'il s'agissait d'une commande de l'éditeur ou si Mme Guellouz s'est elle-même proposée pour l'aventure, mais il semble bien que celle-ci (agrégée de lettres, docteure d'État et professeure à l'Université de Caen) n'ait pas mesuré l'ampleur des défis qui l'attendaient. Guellouz se donne comme objet d'étude, rien de moins que $<<$ l'ensemble des textes où l'échange est systématiquement mis en oeuvre $>>$, et comme but : $<<$ l'élaboration d'une monographie du genre, c'est-à-dire une étude qui, 
tout en prenant en compte le corpus -- immense -- qui s'est constitué à travers le temps et l'espace ou, du moins (...) le plus grand nombre possible de textes, mette en valeur ce qui peut être considéré comme permanent, autrement dit les éléments constitutifs de la forme, sa poétique>> ( 21).

La tâche était herculéenne et on ne peut sans doute reprocher à l'auteure[8] d'en arriver, malgré son érudition impressionnante, à un résultat final qui n'est pas des plus probants. Les problèmes se dessinent d'emblée avec les postulats de départ de Guellouz qui cherche à trouver, comme on vient de le voir, ce qu'il y a de $<<$ permanent $>>$ dans le genre dialogué. Cette quête d'une sorte de poétique atemporelle du dialogue, si elle est discutable, ne me paraît pas en soi absolument problématique, mais les choses se détériorent quand Guellouz cite les écrits qui <<sont à l'origine de [son] travail > $>$ : $<<$ d'une part l'ouvrage de Rudolf Hirzel qui a pour titre Der Dialog, ein literar historischer Versuch, d'autre part, l'article de Roland Mortier qui s'intitule <<Pour une poétique du dialogue : essai de théorie d'un genre>> ( 21).

On ne saurait trouver d'ouvrages plus divergents dans leur approche. Le premier constitue une sorte de survol histo- rique du dialogue, un <<inventaire>> érudit (<<à l'allemande>>) publié en 1895 et consacré surtout au dialogue de l'Antiquité. Le second est un bref article publié en 1984 qui, à la manière des approches poétiques françaises des années soixante-dix, tente de définir certaines caractéristiques formelles du dialogue comme genre (au niveau de l'espace, du temps, des personnages, etc.). Guellouz voit une certaine complémentarité entre ces deux ouvrages, la rigueur $<<$ synchronique $>>$ du second permettant de corriger l'approche $<<$ diachronique $>>$ discutable $<<$ à la manière de Croiset ou de Lanson >> (22) du premier.

La faiblesse de la monographie de Guellouz vient en grande partie de cette double paternité intellectuelle dont elle se réclame avec une fierté pour le moins troublante.[9] Les approches historiques du littéraire ont pourtant quelque peu évolué depuis l'époque de Lanson! Faire fi des nombreuses réflexions, de plus en plus fines et complexes, sur l'historicité du littéraire et croire que l'on pourra $<<$ corriger $>>$ l'historicisme simpliste du début du siècle, en lui juxtaposant une approche formaliste et exclusivement synchronique non moins simpliste, relève de la plus élémentaire innocence!

Partie de tels principes, Guellouz ne peut que reproduire la dichotomie facile du synchronique et du diachronique. Et c'est effectivement ce à quoi elle se consacrera inlassablement dans la plus grande partie de son ouvrage en tentant, d'une part, de définir certaines caractéristiques formelles, générales et atemporelles, du dialogue (dans les première et deuxième parties de la monographie) et en brossant, d'autre part, un tableau 
historique simplement énumératif et descriptif, digne des Lanson et compagnie (dans la troisième partie). Prise entre les Charybde et Scylla de l'historicisme simpliste et du formalisme faussement rigoureux, Guellouz se précipite tour à tour sur l'un et sur l'autre croyant par là échapper aux deux.

Ceci dit, on ne peut pour autant condamner tout l'ouvrage qui demeure fort utile pour toute personne intéressée par cette forme discursive méconnue qui constitue, comme le rappelle à juste titre Guellouz, $<<$ un genre qui est sans doute des plus difficiles à cerner $>>(11)$. Le style un peu vieillot et fort académique de l'auteure ne rend pas la lecture des plus stimulantes, mais l'accumulation de nombreuses citations et de références ouvre tout un champ de possibilités et offre de nombreuses pistes au chercheur qui travaille sur le sujet. L'ouvrage peut également -- faute de mieux! -constituer une introduction au genre du dialogue pour le néophyte (même s'il s'agit d'un texte qui se veut beaucoup plus ambitieux qu'un simple $<<$ Que sais-je? $>>$ ).

J'ajouterais également que certaines distinctions et typologies, bien que parfois discutables (mais quelle typologie ne l'est pas?), permettent de réfléchir aux limites et aux variantes du genre. Dans son introduction, l'auteure énumère par exemple quatre acceptions du terme de dialogue : le $<<$ dialogue-acte $>>$ qui n'est en fait que la conversation réelle et qui renvoie au langage naturel, le $<<$ dialogue-principe $>>$ qui concerne plutôt le $<<$ dialogisme $>>$ tel que défini par Bakhtine ou plus récemment par Francis Jacques et qui relève du registre de $l^{\prime}<<$ abstraction $>>$, le $<<$ dialogue-procédé $>>$ que l'on retrouve dans les récits, les romans ou au théâtre, et enfin le $<<$ dialogue-forme $>>$-- celui qui nous intéresse -- qui est $<<$ autonome sans être indépendant des précédents >>(13-21). Guellouz prend heureusement bien soin de préciser que les frontières entre ces différents avatars du dialogue sont perméables. En effet, on ne doit pas isoler complètement le genre dialogué de son modèle (la conversation naturelle, orale) des principes dialogiques abstraits qui le caractérisent (ou non) ou des procédés dialogaux dans les genres littéraires voisins.

En ce qui concerne le dernier type de dialogue, le $<<$ dialogue forme $>>$, Guellouz introduira de nouvelles distinctions qui mèneront à une typologie tripartie du genre. Après avoir écarté les typologies fondées sur le caractère narratif ou dramatique du dialogue, sur leur sujet, sur leur ton ou encore sur le type de personnages, Guellouz $<<$ se propose de classer les textes selon le statut qu'ils attribuent à l'autre>> (89).

Elle divise d'abord les dialogues en deux catégories, selon que l'autre ne s'y construise <<qu'en fonction de l'un>> ou qu'il <<existe pour lui-même>>. La première catégorie se divise en deux nouveaux types : le dialogue à conversion où le $<<t u>>$ n'est que le double ou relais $d u<<j e>>$ et le 
dialogue pédagogique où le $<<\mathrm{tu}>>$ sert de repoussoir au $<<$ je $>>$. Fait étrange, seul le dialogue pédagogique obtient le privilège d'accéder à la typologie guellouzienne; le dialogue à conversion, qui ressortirait en fait au monologue, se voit déclassé (même s'il se présente sous le $<<$ déguisement $>>$ du dialogue). La deuxième catégorie se divise elle aussi en deux types : le dialogue éristique où $<<$ les deux partenaires incarnent des forces égales qui s'affrontent sans que jamais intervienne la moindre conversion $>>$ et le dialogue heuristique où $<<$ ils coexistent bénéfiquement, sans doute parce que chacun porte en soi sa propre altérité >> (89-90).

La typologie tripartite de Guellouz -- qu'elle reprendra et confirmera dans sa conclusion à l'aide du modèle actantiel greimassien -- implique une forme de gradation dans le degré de $<<$ dialogisme >>, d'ouverture à l'altérité. Le dialogue éristique arrive bon dernier puisqu'il $<<$ remet en cause ce qui est la raison d'être du dialogue, à savoir la possibilité de communiquer. $>>$ (91). Le dialogue pédagogique s'en tire un peu mieux, mais $l^{\prime}<<$ autre $>>$ a le défaut d'y être le plus souvent soumis au $<<$ maître $>>$. Le dialogue heuristique constitue évidemment le type idéal, les interlocuteurs n'étant $<<$ ni tout à fait opposés l'un à l'autre, comme c'est le cas dans le dialogue éristique, ni tout à fait unis, comme c'est le cas dans le dialogue pédagogique, (...) [ils] sont dans cette situation où il ne s'agit plus de transfert d'informations (...) mais de cette $<<$ coopération dans le discours $>>$, de cette $<<$ production conjointe du sens $>>$ (l'auteure reprend ici la distinction qu'établit Francis Jacques entre communicativité et communicabilité).>>

Cette typologie <<évaluative>> me paraît problématique à plusieurs égards. Premièrement, parce qu'il s'agit d'une grille analytique qui tend à exclure certains types, tout à fait légitimes selon moi, de dialogues que l'on ne peut simplement éliminer pour des raisons <<éthico-théoriques >> : les dialogues à conversion par exemple, mais aussi les dialogues satiriques (tels ceux de Lucien) qu'on aurait bien du mal à faire entrer dans une telle typologie. Deuxièmement, parce que fonder une typologie du genre dialogué sur le rapport entre les interlocuteurs, c'est oublier un aspect important de celui-ci : le dialogue écrit est toujours produit par un seul auteur qui ne fait que représenter par écrit un échange entre deux ou plusieurs interlocuteurs (ce dont sont conscients autant Bakhtine que Francis Jacques d'ailleurs). Cela n'implique pas la disqualification automatique de toute approche fondée sur le dialogisme et le rapport à l'altérité, mais devrait nous inciter à tout le moins à une certaine prudence méthodologique. Troisièmement, parce que cette typologie -- comme toute typologie sans doute -- n'est tout simplement pas opératoire. En témoigne le fait que Guellouz choisit comme exemples ultimes de dialogue heuristique les dialogues platoniciens.[10] Or, on pourrait tout aussi pertinemment décrire ceux-ci, dans certains cas, comme des dialogues éristiques (les dialogues aporétiques par exemple) ou même approcher l'ensemble du corpus dialogique platonicien sous l'angle du dialogue pédagogique puisque Socrate y joue habituellement le rôle de maître où il n'est certainement pas dans un rapport de forces <<égales>> avec ses interlocuteurs.[11] 
Mais venons-en maintenant -- et plus rapidement -- au coeur de l'ouvrage. Guellouz divise son étude en trois grandes parties qu'elle intitule respectivement : $<<$ À la recherche d'un genre $>>,<<$ Pour une poétique du genre $>>$ et $<<$ Repères historiques $>>$.

La première partie concerne les définitions et délimitations du genre. Elle comprend un chapitre, qui me paraît d'ailleurs peu utile, sur des genres supposément $<<$ voisins $>>$ du dialogue (négociation, interview, table ronde, théâtre, lettre, discours, traité et essai). Le premier chapitre, par contre, est plus intéressant en ce qu'il fait le relevé des définitions et de la terminologie associées au dialogue. L'auteure y discute notamment des dénominations diverses du genre (colloque, entretien, conversation, etc.) et des nombreuses confusions sémantiques autour du terme de $<<$ dialogue $>>$. Elle montre également à quel point le dialogue a pu échapper aux nombreuses théorisations du littéraire à travers l'histoire (et ce, autant dans les traités de rhétorique et de stylistique, que dans les systèmes typologiques inspirés d'Aristote ou les poétiques contemporaines issues du structuralisme). Elle aurait cependant gagné à étudier davantage les diverses tentatives de systématisation du genre à la Renaissance (Sigonio, Le Tasse, Speroni, Castelvetro, etc.), la seule époque qui ait donné naissance à de véritables tentatives de description du dialogue.

La seconde partie, avec son titre ambitieux et prometteur annonçant une $<<$ poétique du genre $>>$, s'avère plutôt décevante. L'auteure s'attaque à nouveau à la définition (toujours problématique) et à la typologie du dialogue; elle s'attarde ensuite à certains de ses aspects formels (dimension spatio-temporelle, actions et personnages) à partir notamment de Mortier; elle aborde, enfin, l'importante dimension rhétorique du genre. C'est dans cette dernière section surtout que la déception est la plus grande. Elle n'est consacrée, en effet, qu'à la problématique de la représentation de l'oralité dans le dialogue écrit. Si cette dimension du dialogue -- toujours constitué de $<<$ voix imprimées $>>$-- est effectivement essentielle, il aurait cependant fallu développer davantage la question de la rhétorique proprement dite: elle est étroitement liée au genre puisque le dialogue est presque toujours une oeuvre de persuasion.[12] Certains vont même jusqu'à affirmer que le dialogue, en tant que forme discursive, relève beaucoup plus de la rhétorique que de la poétique. Il aurait donc été nécessaire, dans un ouvrage d'une telle envergure, de prendre en compte l'importance de cette dimension (et de son interaction essentielle avec la dialectique) pour la compréhension du dialogue.

Enfin, dans la troisième et dernière partie, Guellouz entreprend un survol historique général de l'évolution du dialogue (en Occident surtout). Cette section est divisée en quatre grandes périodes : l'Antiquité, le Moyen Âge et la Renaissance, les XVIIe et XVIIIe siècles et les XIXe et XXe siècles. Ce survol historique se caractérise malheureusement (mais sans doute 
inévitablement) par une rhétorique de l'énumération et de l'anecdote, impressionnante de par son érudition, mais assez peu substantielle tout compte fait. Il faut cependant rappeler que l'entreprise était on ne peut plus ambitieuse et que Guellouz a le mérite malgré tout de nous donner un des premiers survols -- forcément incomplet -- de l'ensemble de l'histoire du genre.[13]

La conclusion de Guellouz appelle également quelques commentaires. L'auteure semble vouloir corriger son approche jusqu'ici assez $<<$ traditionnelle >> en faisant intervenir à la /pp. 12-13/ toute fin diverses théories contemporaines pour aborder le dialogue sous différents angles, à savoir ses dimensions formelle, psychanalytique, philosophique et historicosociale. Malheureusement, c'est trop peu trop tard. Le recours au modèle actantiel de Greimas n'apporte rien de nouveau (sinon un peu de jargon sémiotique agaçant) à son analyse et les intuitions et hypothèses qu'elle déballe ensuite pêle-mêle sur les aspects psychanalytiques, philosophiques et historiques du dialogue, bien que parfois intéressantes, sont trop peu développées pour être vraiment significatives. Il aurait fallu s'y mettre plus tôt.

Je ne pourrais conclure sans mentionner une autre faiblesse -- plus grave celle-là -- de la monographie de Guellouz. Elle réside dans ce qu'on pourrait appeler son $<<$ latino-centrisme $>>$ (qui prend même parfois la forme plus précise d'un agaçant $<<$ gallocentrisme $>>$ ). Autant le choix du corpus que les références théoriques témoignent en effet de ce parti-pris national et linguistique pour les cultures latines ( plus particulièrement celles de la France, de l'Espagne et de l'Italie, sans trop de mention de la tradition portugaise). En ce qui concerne par exemple le corpus, Guellouz ne se gêne pas pour justifier un certain <<déséquilibre>> (sic) dans le choix des textes:

D'où encore, dans notre travail, un certain déséquilibre - -- s'agissant des textes étudiés -- entre les différentes aires linguistiques. Ce déséquilibre s'explique par le fait que seuls les Italiens, les Espagnols et surtout les Français (re-sic) ont fait délibérément sortir le dialogue du domaine de la philosophie pure. (23)

Cette affirmation est absolument sans fondement (sauf, peut-être, pour la période de l'impérialisme culturel français des XVIIe et XVIIIe siècles). Pour la Renaissance seulement (période que je connais plus particulièrement), Roger Lee Deakins[14] compte 56 dialogues entre 1500 et 1558 en Angleterre, alors que John T. Day[15] en dénombre 206 entre 1558 et 1603. Et il ne s'agit pas le moins du monde de dialogues de $<<$ philosophie pure $>>$ comme en témoigne entre autres les dialogues de Roger Ascham, de Thomas Elyot (jamais cités par Guellouz) ou encore de Thomas More (dont elle ne 
mentionne qu'un seul[16] -- et le moins intéressant - -- des importants dialogues). Pour la Renaissance toujours, il aurait fallu mentionner également les dialogues allemands de Hutten ou les nombreux dialogues portugais de l'époque. À la décharge de l'auteure, on peut toujours croire qu'elle a été mal servie par ses sources puisqu'elle se sert pour le dialogue anglais du seul livre -- très daté et franchement mauvais -- d'Elisabeth Merril.[17]

On peut faire l'hypothèse que Guellouz ne lit pas l'anglais, car son manque d'intérêt pour la tradition dialogique anglo-saxonne se double d'une ignorance complète des recherches récentes publiées en anglais dans le domaine du dialogue. Cet <<oubli > > remarquable -- et impardonnable à une époque où les recherches bibliographiques sont devenues tellement aisées -- contribue à faire vieillir prématurément cette monographie qui se voit privée de l'apport de certaines des meilleures études des dernières décennies sur le genre. Je songe notamment -- pour la Renaissance seulement! -- au brillant livre de Jon Snyder sur les poétiques du dialogue à la Renaissance, ou aux livres de David Marsh sur le dialogue italien du Quattrocento et de Kenneth Wilson sur le dialogue anglais de la période Tudor, voire même aux recherches plus anciennes (mais encore essentielles) de C.J.R. Armstrong et du père Ong sur les rapports entre dialogue et dialectique.[18]

La monographie de Guellouz aurait certainement gagné à s'abreuver à ces sources, et l'approche plus fine et plus informée d'un Snyder par exemple lui aurait peut-être permis d'éviter les écueils de l'historiographie littéraire traditionnelle et du formalisme abstrait un peu scolaire sur lesquels elle bute constamment. Ceci dit, je n'oserais pas pour autant condamner l'ouvrage dans son ensemble. L'érudition de Guellouz fait tout de même de sa monographie une source inépuisable d'informations, de références et de citations sur le dialogue. Et elle ouvre au moins la voie à un dialogue épistémologique renouvelé sur ce genre trop peu connu et étudié.

\author{
Jean-François Vallée
}

Département de Littérature comparée

\author{
Université de Montréal
}


[1]Il n'en existe d'ailleurs pas non plus, à ma connaissance, dans d'autres langues occidentales. Je n'ai jamais du moins rencontré d'ouvrage vraiment général sur le dialogue ni en anglais, ni en espagnol, ni en allemand.

[2]Collection dirigée actuellement par Robert Mauzi et qui comprend déjà plusieurs monographies sur différents mouvements littéraires (surréalisme, naturalisme, maniérisme, romantisme, symbolisme, etc.) ainsi que sur différents genres (roman, théâtre, roman épistolaire, tragédie classique, journal intime, épopée, biographie, etc.)

[3]Pour le dialogue antique en général : Jean Andrieu, Le dialogue antique structure et présentation, Les Belles Lettres, Paris, 1954; pour le dialogue platonicien : Jean Laborderie, Le dialogue platonicien de la maturité, Les Belles Lettres, Paris, 1978; pour le dialogue romain en général : Pierre Grimal, "Caractères généraux du dialogue romain. De Lucilius à Cicéron" in L'Information Littéraire 5, Paris, nov.-déc. 1955; pour le dialogue cicéronien : Michel Ruch, Le préambule dans les oeuvres philosophiques de Cicéron. Essai sur la genèse et l'art du dialogue, Publications de la Faculté de lettres de l'université de Strasbourg, Les Belles Lettres, Paris, 1958, etc.

[4]Notamment l'ouvrage de Jean-Claude Aubailly, Le monologue, le dialogue et la sottie. Essai sur quelques genres dramatiques à la fin du moyen âge et au début du 16e siècle, Champion, Paris, 1975.

[5]Tels que, entre autres, la monographie de Mustapha Kemal Bénouis, Le dialogue philosophique dans la littérature française du seizième siècle, Mouton, The Hague -- Paris, 1976, ou les nombreux articles d'Eva Kushner dont on peut citer, par exemple, "Le dialogue en France de 1550 à 1560" publié dans un collectif entièrement consacré au dialogue renaissant : Le dialogue au temps de la Renaissance, dir. M. T. Jones-Davies, Centre de recherches sur la Renaissance, Jean Touzot Libraire-éditeur, Paris, 1984.

[6]Parmi lesquels on peut citer notamment les études de Bernard Beugnot (L'entretien au XVIIe siècle, Leçon inaugurale, Université de Montréal, 17 février 1971) et Bernard Bray ("Le dialogue comme forme littéraire au XVIIe siècle", Cahiers de l'Association internationale des Etudes françaises, no 24, mai 1972) pour le XVIIe ou de Maurice Roelens ("Le dialogue philosophique, genre impossible? L'opinion des siècles classiques", Cahiers de l'Association internationale des Etudes françaises, no 24, mai 1972) et Roland Mortier ("Variations on the Dialogue of the French Enligthenment", Studies in Eigtheenth Century Culture, vol. 16, Cleveland, Ohio, 1986) pour le XVIIIe. Sans compter les nombreuses études ponctuelles de dialoguistes tels Diderot, Sade, Fontenelle, Bouhours, Guez de Balzac, etc.

[7]Je n'en connais que deux : celui de Roland Mortier, "Pour une poétique du dialogue : essai de théorie d'un genre" in Literary Theory and Criticism, Festschrift presented to René Wellek in honor of his eightieth birthday, P. Lang, Berne, 1984; et celui de Michel Le Guern, "Sur le genre du dialogue", in L'Automne de la Renaissance, Paris, 1981. 
[8]Précisons, pour les lecteurs français que l'orthographie "auteure" est maintenant répandue et acceptée au Québec.

[9]D'ailleurs, comme si elle s'était par la suite rendue compte de l'absurdité de cette paternité bicéphale, Guellouz ne fera pas vraiment appel à ses deux "mentors" dans son ouvrage : l'index des auteurs nous apprend en effet qu'hormis leur mention dans la pétition de principe de l'introduction, les deux auteurs ne sont cités que deux fois chacun.

[10]Les deux autres exemples de dialogues heuristiques réussis sont Le neveu de Rameau de Diderot et l'Heptalomeres de Bodin.

[11]J'ajouterais également que Guellouz, en établissant sa typologie, oublie de mentionner l'apport important d'Eva Kushner qui vingt ans plus tôt analysait déjà -- et peut-être avec plus de circonspection -- les dialogues de la Renaissance à partir de ces catégories du dialogique et du rapport à l'altérité.

[12]John McClelland parle du dialogue comme d'une "rhétorique à deux temps" : "Le dialogue est un rhétorique à deux temps. D'abord il mime les tentatives des interlocuteurs de se persuader mutuellement de la justesse de leurs positions. Sur ce plan il témoigne des arguments contemporains et de leur efficacité relative. Mais en tant que littérature du type "essai" ou "nonfiction", il vise l'instruction d'un public et son adhésion à un point de vue particulier. (...) Le véritable intérêt du dialogue n'est pas que le genre capte la spontanéité de la conversation -- cela n'est qu'une fiction alléchante -mais plutôt que la représentation du jeu réciproque de la conversation mime immédiatement et efficacement l'imprévisible de la pensée (...). La rhétorique du dialogue consiste à exposer en détail l'acheminement logique d'une intelligence qui recherche à propos d'une question sérieuse une position conséquente et presque inévitable. Le lecteur sera persuadé autant par la mimésis du procédé que par les arguments eux-mêmes."

John McClelland, "Dialogue et rhétorique à la Renaissance" in Le dialogue, dir. P. R. Léon et P. Perron, Didier-Presses de l'Université d'Ottawa, Ottawa, 1985.

[13]Sans compter, évidemment, l'ouvrage de Hirzel.

[14]Roger Lee Deakins, The Tudor Dialogue as a Literary Form, thèse de doctorat, Harvard University, 1964.

[15]John T. Day, Elizabethan Prose Dialogues, thèse de doctorat, Harvard University, 1977. Le caractère très incomplet de l"Index" de Bénouis est démontré ici dans le fait qu'il n'avait trouvé, quant à lui, que 5 dialogues anglais pour tout le XVIe siècle!

[16]Il s'agit du Dialogue Concerning Heresies beaucoup moins important que le dialogue du premier livre de l'Utopie ou le Dialogue of Comfort against Tribulation) 
[18]Quelques exemples d'excellentes études du dialogue publiées en anglais : C.J.R. Armstrong, "The Dialectical Road to Truth : The Dialogue" in French Renaissance Studies, 1540-1570, P. Sharratt ed., Edinburgh University Press, Edinburgh, 1976 ; David Marsh, The Quattrocento Dialogue, Harvard University Press, Cambridge, 1980 ; Walter J. Ong, Ramus : Method, and the Deacy of Dialogue. From the Art of Discourse to the Art of Reason, Harvard University Press, Cambridge, 1958 ; Jon R. Snyder, Writing the Scene of Speaking: Theories of Dialogue in Later Italian Renaissance, Stanford University Press, Stanford, 1989 ; Kenneth J. Wilson, Incomplete Fictions. The Formation of English Renaissance Dialogue, The Catholic University of America Press, Washington, 1985 ou, du même auteur, The Continuity of Post-Classical Dialogue" in Cithara, 21, 1981. 